PROCEEDINGS OF THE

AMERICAN MATHEMATICAL SOCIETY

Volume 129, Number 6, Pages 1721-1726

S 0002-9939(00)05691-4

Article electronically published on November 2, 2000

\title{
HILBERT MODULES OVER A CLASS OF SEMICROSSED PRODUCTS
}

\author{
DALE R. BUSKE
}

(Communicated by David R. Larson)

\begin{abstract}
Given the disk algebra $\mathcal{A}(\mathbb{D})$ and an automorphism $\alpha$, there is associated a non-self-adjoint operator algebra $\mathbb{Z}^{+} \times{ }_{\alpha} \mathcal{A}(\mathbb{D})$ called the semicrossed product of $\mathcal{A}(\mathbb{D})$ with $\alpha$. Buske and Peters showed that there is a one-to-one correspondence between the contractive Hilbert modules $\mathcal{H}$ over $\mathbb{Z}^{+} \times_{\alpha} \mathcal{A}(\mathbb{D})$ and pairs of contractions $S$ and $T$ on $\mathcal{H}$ satisfying $T S=S \alpha(T)$. In this paper, we show that the orthogonally projective and Shilov Hilbert modules $\mathcal{H}$ over $\mathbb{Z}^{+} \times_{\alpha} \mathcal{A}(\mathbb{D})$ correspond to pairs of isometries on $\mathcal{H}$ satisfying $T S=S \alpha(T)$. The problem of commutant lifting for $\mathbb{Z}^{+} \times{ }_{\alpha} \mathcal{A}(\mathbb{D})$ is left open, but some related results are presented.
\end{abstract}

\section{INTRODUCTION}

In an effort to develop for an $n$-tuple of commuting contractions a theory analogous to the famous model theory of Sz. Nagy and Foias $[\mathrm{Sz}-\mathrm{NF}]$ for a single contraction, Douglas and Paulsen $[\mathrm{DP}]$ reformulated much of dilation theory in the context of Hilbert modules. A class of "nice" modules, the so-called Shilov modules, was introduced and contractive Hilbert modules were studied via their Shilov resolutions. These Shilov resolutions are tightly connected to Arveson's dilation theorem Arv. Douglas and Paulsen also related the notion of commutant lifting Sz-NF to the concept of "hypoprojective" modules. However, it seemed that neither of these types of modules was the proper operator algebraic analogue of a projective module in homological algebra. Recently, Muhly and Solel [MS] have introduced the concept of orthogonally projective Hilbert modules in an effort to better understand the concept of "hypoprojective" (henceforth called strongly orthogonally projective as in [MS]) Hilbert modules. We study all of these Hilbert modules over semicrossed products in the hope that it may aid in the development of the proper operator algebraic analogue of projective modules.

A semicrossed product of the disk algebra, denoted $\mathbb{Z}^{+} \times_{\alpha} \mathcal{A}(\mathbb{D})$, is a non-selfadjoint operator algebra associated to the pair $(\mathcal{A}(\mathbb{D}), \alpha)$ where $\alpha$ is an automorphism of the disk algebra $\mathcal{A}(\mathbb{D})$. Its construction is similar to that of its $C^{*}$ envelope-the full crossed product $\mathbb{Z} \times{ }_{\alpha} C(\mathbb{T})[\mathrm{BP}$. Furthermore, the contractive representations of $\mathbb{Z}^{+} \times_{\alpha} \mathcal{A}(\mathbb{D})$ on a Hilbert space $\mathcal{H}$ are known to be completely

Received by the editors June 23, 1998 and, in revised form, September 17, 1999.

2000 Mathematics Subject Classification. Primary 47H20, 46M18; Secondary 47A15, 47A45, $47 \mathrm{~A} 56$.

Key words and phrases. Commutant lifting, Hilbert modules, Shilov modules, orthogonally projective modules. 
contractive and to correspond to pairs of contractions $S$ and $T$ on $\mathcal{H}$ satisfying $T S=S \alpha(T)$. The main result of Section 2, Theorem 2.3] characterizes the Shilov and orthogonally projective Hilbert modules $\mathcal{P}$ over $\mathbb{Z}^{+} \times{ }_{\alpha} \mathcal{A}(\mathbb{D})$ as those corresponding to pairs of isometries $S$ and $T$ on $\mathcal{P}$ satisfying $T S=S \alpha(T)$. Sections 3 and 4 investigate further these Shilov modules.

\section{SHILOV MODULES}

Let $\mathfrak{A}$ be an operator algebra with $C^{*}$-envelope $C^{*}(\mathfrak{A})$. A Hilbert module $\mathcal{H}$ for $\mathfrak{A}$ is Shilov if there exists a $C^{*}(\mathfrak{A})$-module $\mathcal{K}$ such that $\mathcal{H}$ is isomorphic to a submodule of $\mathcal{K}$ viewed as an $\mathfrak{A}$-module. A sequence of Hilbert modules over $\mathfrak{A}$,

$$
0 \rightarrow \mathcal{K} \stackrel{\Psi}{\rightarrow} \mathcal{M} \stackrel{\Phi}{\rightarrow} \mathcal{H} \rightarrow 0,
$$

is a short exact isometric sequence if $\mathcal{K}$ is isomorphic to a submodule of $\mathcal{M}$ having quotient isomorphic to $\mathcal{H}$. A Hilbert module $\mathcal{H}$ over $\mathfrak{A}$ admits a Shilov resolution if $\mathcal{K}$ and $\mathcal{M}$ are Shilov modules and $\Psi$ and $\Phi$ are bounded module maps in (1). If such a resolution (11) exists, then $(\mathcal{M}, \Phi)$ is called a Shilov dominant for $\mathcal{H}$. A Shilov dominant $(\mathcal{M}, \Phi)$ for $\mathcal{H}$ is strongly minimal if there is no submodule $\mathcal{N}$ of $\mathcal{M}$ such that $\Phi$ maps $\mathcal{N}$ coisometrically onto $\mathcal{H}$.

For the semicrossed products $\mathbb{Z}^{+} \times{ }_{\alpha} \mathcal{A}(\mathbb{D})$, every contractive Hilbert module $\mathcal{H}$ is completely contractive [BP, Corollary III.14] so that $\mathcal{H}$ admits a Shilov resolution Arv. The purpose of this section is to characterize the Shilov modules over $\mathbb{Z}^{+} \times_{\alpha}$ $\mathcal{A}(\mathbb{D})$. To this end, we utilize the notion of an orthogonally projective module first introduced by Muhly and Solel [MS].

A contractive Hilbert module $\mathcal{P}$ over an operator algebra $\mathfrak{A}$ is orthogonally projective if every short exact isometric sequence

$$
0 \rightarrow \mathcal{K} \rightarrow \mathcal{M} \stackrel{\Phi}{\rightarrow} \mathcal{P} \rightarrow 0
$$

ending at $\mathcal{P}$ is split by a contraction. That is, there exists a module map $\widetilde{\Phi}: \mathcal{P} \rightarrow \mathcal{M}$ with $\|\widetilde{\Phi}\| \leq 1$ and $\Phi \circ \widetilde{\Phi}=1_{\mathcal{P}}$. A method of characterizing orthogonally projective modules is given in Lemma 2.1

Let $\mathcal{P}$ and $\mathcal{K}$ be contractive Hilbert modules over an operator algebra $\mathfrak{A}$. A $(\mathcal{P}, \mathcal{K})$-derivation is a continuous complex linear map $D: \mathfrak{A} \rightarrow B(\mathcal{P}, \mathcal{K})$ such that $D(F G)=D(F) \rho_{\mathcal{P}}(G)+\rho_{\mathcal{K}}(F) D(G)$ for all $F, G \in \mathfrak{A}$.

Lemma 2.1 ([MS, Proposition 2.6]). Let (2) be a short exact isometric sequence of contractive Hilbert modules. The representation $\rho_{\mathcal{M}}$ is unitarily equivalent to

$$
F \longmapsto\left(\begin{array}{cc}
\rho_{\mathcal{K}}(F) & D(F) \\
0 & \rho_{\mathcal{P}}(F)
\end{array}\right)
$$

for some $(\mathcal{P}, \mathcal{K})$-derivation $D$. If the only $(\mathcal{P}, \mathcal{K})$-derivation for which this occurs is $D=0$, then $\mathcal{P}$ is orthogonally projective.

Recall that if $\mathcal{P}$ is a contractive Hilbert module over $\mathbb{Z}^{+} \times{ }_{\alpha} \mathcal{A}(\mathbb{D})$, where $\alpha(f)(z)=$ $f(\varphi(z))$ for some Möbius transformation $\varphi$ of $\mathbb{D}$, then $S_{\mathcal{P}} \equiv \rho_{\mathcal{P}}\left(\delta_{1} \otimes 1\right)$ and $T_{\mathcal{P}} \equiv \rho_{\mathcal{P}}\left(\delta_{0} \otimes z\right)$ are contractions satisfying the relation $T_{\mathcal{P}} S_{\mathcal{P}}=S_{\mathcal{P}} \varphi\left(T_{\mathcal{P}}\right)$ BP Theorem III.10].

Lemma 2.2. Let $\mathcal{P}$ be a contractive Hilbert module over $\mathbb{Z}^{+} \times_{\alpha} \mathcal{A}(\mathbb{D})$. If $S_{\mathcal{P}} \equiv$ $\rho_{\mathcal{P}}\left(\delta_{1} \otimes 1\right)$ and $T_{\mathcal{P}} \equiv \rho_{\mathcal{P}}\left(\delta_{0} \otimes z\right)$ are isometries, then $\mathcal{P}$ is orthogonally projective. 
Proof. Let (2) be a short exact isometric sequence of contractive Hilbert modules ending at $\mathcal{P}$. Let $D$ be a $(\mathcal{P}, \mathcal{K})$-derivation such that $\rho_{\mathcal{M}}$ is unitarily equivalent to the representation $\rho$ on $\mathcal{K} \oplus \mathcal{P}$ given by (3). By Lemma 2.1, we need only show that $D \equiv 0$ for it to follow that $\mathcal{P}$ is orthogonally projective. Since $\mathcal{P}, \mathcal{M}$, and $\mathcal{K}$ are contractive Hilbert modules and $\rho_{\mathcal{M}}$ is unitarily equivalent to $\rho$, it follows that

$$
\left(\begin{array}{cc}
\rho_{\mathcal{K}}\left(\delta_{0} \otimes z\right) & D\left(\delta_{0} \otimes z\right) \\
0 & \rho_{\mathcal{P}}\left(\delta_{0} \otimes z\right)
\end{array}\right) \quad \text { and } \quad\left(\begin{array}{cc}
\rho_{\mathcal{K}}\left(\delta_{1} \otimes 1\right) & D\left(\delta_{1} \otimes 1\right) \\
0 & \rho_{\mathcal{P}}\left(\delta_{1} \otimes 1\right)
\end{array}\right)
$$

are contractions. Since $T_{\mathcal{P}}$ is an isometry,

$$
\begin{aligned}
& \left\|\left(\begin{array}{cc}
\rho_{\mathcal{K}}\left(\delta_{0} \otimes z\right) & D\left(\delta_{0} \otimes z\right) \\
0 & T_{\mathcal{P}}
\end{array}\right)\right\|^{2} \\
& =\left\|\left(\begin{array}{cc}
* & * \\
* & D\left(\delta_{0} \otimes z\right)^{*} D\left(\delta_{0} \otimes z\right)+T_{\mathcal{P}}^{*} T_{\mathcal{P}}
\end{array}\right)\right\| \\
& \geq\left\|D\left(\delta_{0} \otimes z\right)^{*} D\left(\delta_{0} \otimes z\right)+I_{\mathcal{P}}\right\|>1
\end{aligned}
$$

if $D\left(\delta_{0} \otimes z\right) \neq 0$. Similarly,

$$
\left\|\left(\begin{array}{cc}
\rho_{\mathcal{K}}\left(\delta_{1} \otimes 1\right) & D\left(\delta_{1} \otimes 1\right) \\
0 & S_{\mathcal{P}}
\end{array}\right)\right\|^{2}>1
$$

if $D\left(\delta_{1} \otimes 1\right) \neq 0$. Hence, $D\left(\delta_{0} \otimes z\right)=0=D\left(\delta_{1} \otimes 1\right)$. Since $D$ is a derivation, $D\left(\sum_{i=0}^{n} \delta_{i} \otimes p_{i}\right)=0$ for all polynomials $p_{i}$ and hence $D \equiv 0$ by continuity.

Theorem 2.3. Let $\mathcal{P}$ be a contractive Hilbert module over $\mathbb{Z}^{+} \times{ }_{\alpha} \mathcal{A}(\mathbb{D})$ determined by $S_{\mathcal{P}} \equiv \rho_{\mathcal{P}}\left(\delta_{1} \otimes 1\right)$ and $T_{\mathcal{P}} \equiv \rho_{\mathcal{P}}\left(\delta_{0} \otimes z\right)$. Then, the following are equivalent:

(i) $S_{\mathcal{P}}$ and $T_{\mathcal{P}}$ are isometries satisfying $T_{\mathcal{P}} S_{\mathcal{P}}=S_{\mathcal{P}} \varphi\left(T_{\mathcal{P}}\right)$.

(ii) $\mathcal{P}$ is an orthogonally projective Hilbert module for $\mathbb{Z}^{+} \times_{\alpha} \mathcal{A}(\mathbb{D})$.

(iii) $\mathcal{P}$ is a Shilov module for $\mathbb{Z}^{+} \times_{\alpha} \mathcal{A}(\mathbb{D})$.

Proof. That (i) $\Rightarrow$ (ii) follows from Lemma [2.2. For (ii) $\Rightarrow$ (iii), since $\mathcal{P}$ is contractive, it is completely contractive [BP, Corollary III.14] so that $\mathcal{P}$ is Shilov [MS, Proposition 3.2]. We show (iii) $\Rightarrow$ (i). Since the $C^{*}$-envelope of $\mathbb{Z}^{+} \times_{\alpha} \mathcal{A}(\mathbb{D})$, $C^{*}\left(\mathbb{Z}^{+} \times_{\alpha} \mathcal{A}(\mathbb{D})\right)$, is the full crossed product $\mathbb{Z} \times{ }_{\alpha} C(\mathbb{T})$ BP, Proposition III.13] and $\mathcal{P}$ is Shilov, there exists a module $\mathcal{M}$ over $\mathbb{Z} \times{ }_{\alpha} C(\mathbb{T})$ such that $\mathcal{P}$ is isomorphic to a submodule of $\mathcal{M}$ where $\mathcal{M}$ is viewed as a module over $\mathbb{Z}^{+} \times{ }_{\alpha} \mathcal{A}(\mathbb{D})$. We investigate the modules $\mathcal{M}$ over $\mathbb{Z} \times{ }_{\alpha} C(\mathbb{T})$; that is, the $C^{*}$-representations $\rho_{\mathcal{M}}$ of $\mathbb{Z} \times{ }_{\alpha} C(\mathbb{T})$ on $\mathcal{M}$. But, the $C^{*}$-representations $\rho_{\mathcal{M}}$ of $\mathbb{Z} \times{ }_{\alpha} C(\mathbb{T})$ on $\mathcal{M}$ correspond bijectively to the covariant representations of $(C(\mathbb{T}), \mathbb{Z}, \alpha)$ on $\mathcal{M}$. These covariant representations $(\pi, U)$ of $(C(\mathbb{T}), \mathbb{Z}, \alpha)$ on $\mathcal{M}$ in turn correspond to pairs of unitaries $V \equiv \pi(z)$ and $U$ on $\mathcal{M}$ satisfying $V U=U \varphi(V)$. Hence, $\rho_{\mathcal{M}}$ is determined by two unitaries $V \equiv \rho_{\mathcal{M}}\left(\delta_{0} \otimes z\right)$ and $U \equiv \rho_{\mathcal{M}}\left(\delta_{1} \otimes 1\right)$ satisfying $V U=U \varphi(V)$. Thus, $\rho_{\mathcal{P}}$ is determined by two isometries $\left.\rho_{\mathcal{P}}\left(\delta_{0} \otimes z\right) \equiv V\right|_{\mathcal{P}}$ and $\left.\rho_{\mathcal{P}}\left(\delta_{1} \otimes 1\right) \equiv U\right|_{\mathcal{P}}$.

Example 2.4. Let $U$ be the unilateral shift on $\bigoplus_{0}^{\infty} L^{2}(\mathbb{T})$ and for $f \in \mathcal{A}(\mathbb{D})$, define $D_{f}\left(\xi_{0}, \xi_{1}, \xi_{2}, \ldots\right)=\left(f \xi_{0}, \alpha(f) \xi_{1}, \alpha^{2}(f) \xi_{2}, \ldots\right)$. The map $\rho: \mathbb{Z}^{+} \times_{\alpha} \mathcal{A}(\mathbb{D}) \rightarrow$ $B\left(\bigoplus_{0}^{\infty} L^{2}(\mathbb{T})\right)$ defined by $\rho\left(\delta_{1} \otimes 1\right)=U$ and $\rho\left(\delta_{0} \otimes f\right)=D_{f}$ is then a contractive representation [BP, Theorem III.10]. By Theorem $2.3, \bigoplus_{0}^{\infty} L^{2}(\mathbb{T})$ is a Shilov module.

Example 2.5. Suppose $\alpha(f)(z)=f(\mu z)$ where $|\mu|=1$ is irrational. Let $C_{\bar{\mu}}$ denote the composition operator defined by $C_{\bar{\mu}}(g(z))=g(\bar{\mu} z)$. Define a contractive 
representation $\rho$ of $\mathbb{Z}^{+} \times_{\alpha} \mathcal{A}(\mathbb{D})$ on $H^{2}(\mathbb{D})$ by $\rho\left(\delta_{1} \otimes 1\right)=C_{\bar{\mu}}$ and $\rho\left(\delta_{0} \otimes f\right)=T_{f}$, the Toeplitz operator $\left[\mathrm{BP}\right.$, Theorem III.10]. By Theorem [2.3, $H^{2}(\mathbb{D})$ is Shilov.

Example 2.6. Suppose $\alpha$ is an irrational rotation as in Example 2.5. Let $\left\{e_{i, j}: i \geq 0\right.$ and $\left.j \geq 0\right\}$ be an orthonormal basis for a Hilbert space $\mathcal{H}$. Define isometries $S$ and $T$ on $\mathcal{H}$ by $S e_{i, j}=e_{i+1, j}$ and $T e_{i, j}=\mu^{i} e_{i, j+1}$. Defining a contractive representation $\rho$ of $\mathbb{Z}^{+} \times_{\alpha} \mathcal{A}(\mathbb{D})$ on $\mathcal{H}$ by $\rho\left(\delta_{1} \otimes 1\right)=S$ and $\rho\left(\delta_{0} \otimes z\right)=T$ makes $\mathcal{H}$ into a Shilov module.

\section{A strongly minimal Shilov dominant}

It is not known whether commutant lifting in the sense of Muhly and Solel MS holds for $\mathbb{Z}^{+} \times{ }_{\alpha} \mathcal{A}(\mathbb{D})$ even in the case where $\alpha$ is a nonperiodic rotation. We assume henceforth that $\alpha(f)(z)=f(\mu z)$ where $|\mu|=1$ is irrational. As a starting point in determining whether commutant lifting holds for this algebra, one would like examples of strongly minimal Shilov dominants. The following exhibits one such example.

Define $\mathfrak{B}_{\alpha}$ to be the norm closed subalgebra of $B\left(H^{2}(\mathbb{D})\right)$ generated by the Toeplitz operators $T_{f}(f \in \mathcal{A}(\mathbb{D}))$ and the composition operator $C_{\bar{\mu}}$. Recall from Buske and Peters $\left[\mathrm{BP}\right.$ Proposition IV.2] that $\mathbb{Z}^{+} \times_{\alpha} \mathcal{A}(\mathbb{D})$ is completely isometrically isomorphic to $\mathfrak{B}_{\alpha}$.

Lemma 3.1. For an integer $N \geq 0$, the nontrivial invariant subspaces $\mathcal{M}$ of $z^{N} H^{2}(\mathbb{D})$ under $\mathfrak{B}_{\alpha}$ are precisely those of the form $\mathcal{M}=z^{m_{0}} H^{2}(\mathbb{D})$ for $m_{0} \geq N$.

Proof. Note that $\mathfrak{B}_{\alpha}$ is the norm closed subalgebra of $B\left(H^{2}(\mathbb{D})\right)$ generated by the Toeplitz operator $T_{z}$ and composition operator $C_{\bar{\mu}}$. Denote by $\mathfrak{B}_{s c}$ the strongly closed algebra generated by the Toeplitz operator $T_{z}$ and composition operator $C_{\bar{\mu}}$. Let $(0) \neq \mathcal{M} \subseteq z^{N} H^{2}(\mathbb{D})$ be a closed invariant subspace under $\mathfrak{B}_{\alpha}$. Then $\mathcal{M}$ is also invariant under $\mathfrak{B}_{s c}$. To see this, note that if $f \in \mathcal{M}$ and $A \in \mathfrak{B}_{s c}$, then there exists a sequence $\left\{A_{n}\right\}_{n=1}^{\infty} \subseteq \mathfrak{B}_{\alpha}$ such that $\left\|A_{n} f-A f\right\| \rightarrow 0$. But, $A_{n} f \in \mathcal{M}$ for $n \geq 0$ and $\mathcal{M}$ closed show that $A f \in \mathcal{M}$.

As in Nordgren, Rosenthal, and Wintrobe [NRW], we show that $\mathfrak{B}_{s c}$ contains the projections $P_{m}: H^{2}(\mathbb{D}) \rightarrow H^{2}(\mathbb{D})$ defined by $\sum_{0}^{\infty} a_{n} z^{n} \mapsto a_{m} z^{m}$ for each $m \in \mathbb{N}$. Define $C_{m}=\bar{\mu}^{-m} C_{\bar{\mu}}$ and $A_{k}=\frac{1}{k} \sum_{j=1}^{k} C_{m}^{j}$ for $k \geq 1$. Let

$$
S=\left\{f \in H^{2}(\mathbb{D}): \lim _{k \rightarrow \infty}\left(A_{k}-P_{m}\right) f=0\right\} .
$$

By the ergodic theorem, $\lim _{k \rightarrow \infty}\left(A_{k}-P_{m}\right) z^{i}=0$ for all $i \geq 0$. Hence, $S=H^{2}(\mathbb{D})$. It follows that $P_{m} \mathcal{M} \neq(0)$ for some $m \geq N$ and hence that $\mathcal{M}$ contains $z^{m}$ for some $m \geq N$. Define $m_{0}=\inf _{m \geq N}\left\{m: z^{m} \in \mathcal{M}\right\}$. Note that $z^{m_{0}} \in \mathcal{M}$ and $z^{m_{0}+k} \in \mathcal{M} \forall k \in \mathbb{N}$ by the invariance of $\mathcal{M}$ under $T_{z}$. Since $\mathcal{M}$ is a closed subspace of $z^{N} H^{2}(\mathbb{D})$ invariant under $T_{z}$ and $m_{0} \geq N$, it is clear that $z^{m_{0}} H^{2}(\mathbb{D}) \subseteq$ $\mathcal{M}$. Let $f(z)=z^{N} \sum_{0}^{\infty} a_{n} z^{n} \in \mathcal{M}$. Then $P_{k}(f(z))=a_{k-N} z^{k} \in \mathcal{M} \forall k \geq$ $N$. Hence $a_{n}=0$ for $0 \leq n<m_{0}-N$ by the definition of $m_{0}$ and $f(z)=$ $z^{N} \sum_{n=m_{0}-N}^{\infty} a_{n} z^{n}=z^{m_{0}} \sum_{n=0}^{\infty} a_{n+m_{0}-N} z^{n} \in z^{m_{0}} H^{2}(\mathbb{D})$. Thus $\mathcal{M} \subseteq z^{m_{0}} H^{2}(\mathbb{D})$ yielding $\mathcal{M}=z^{m_{0}} H^{2}(\mathbb{D})$.

Example 3.2. It is easy to verify that if $\left|z_{0}\right| \leq 1$ and $\xi_{0}=0$ or if $\left|\xi_{0}\right| \leq 1$ and $z_{0}=0$, then $\gamma_{z_{0}}^{\left(\xi_{0}\right)}: \mathfrak{B}_{\alpha} \rightarrow \mathbb{C}$ defined by $C_{\bar{\mu}} \mapsto \xi_{0}$ and $T_{f(z)} \mapsto f\left(z_{0}\right)$ is a character. 
In fact, the character space $\Gamma$ of $\mathfrak{B}_{\alpha}$ is given by

$$
\Gamma=\left\{\gamma_{z_{0}}^{\left(\xi_{0}\right)}:\left|z_{0}\right| \leq 1,\left|\xi_{0}\right| \leq 1, \text { and either } z_{0}=0 \text { or } \xi_{0}=0\right\}
$$

and coincides with the maximal ideal space [BP, Corollary III.11].

Consider then the Shilov resolution of $\mathfrak{B}_{\alpha}$ given by

$$
0 \longrightarrow H_{0}^{2}(\mathbb{D}) \longrightarrow H^{2}(\mathbb{D}) \stackrel{\Phi}{\longrightarrow} \mathbb{C}_{(0,0)} \longrightarrow 0
$$

where $H_{0}^{2}(\mathbb{D})=\left\{\xi \in H^{2}(\mathbb{D}): \xi(0)=0\right\}, \Phi(\xi)=\xi(0)$, and $\mathbb{C}_{(0,0)}$ is the one dimensional Hilbert module $\mathbb{C}$ determined by the character $\gamma_{0}^{(0)}$. That this is a Shilov resolution follows since $H^{2}(\mathbb{D})$ is seen to be a Shilov module for $\mathfrak{B}_{\alpha}$ by Theorem 2.3 . By Lemma 3.1 it follows that $H^{2}(\mathbb{D})$ is strongly minimal for $\mathbb{C}_{(0,0)}$.

For the bidisk algebra $\mathcal{A}\left(\mathbb{D}^{2}\right)$, Example 3.20 of Douglas and Paulsen [DP] gives two strongly minimal Shilov resolutions of $\mathbb{C}$ which are not isomorphic (proving that commutant lifting does not hold for $\mathcal{A}\left(\mathbb{D}^{2}\right)$ ). This should be compared to Example 3.2

\section{An InVARIANT SUBSPACE PROBLEM}

Classifying all the Shilov modules for $\mathbb{Z}^{+} \times{ }_{\alpha} \mathcal{A}(\mathbb{D})$ is quite difficult. This is closely tied to finding a model for a pair of commuting isometries. However, Lemma 3.1 does classify the Hilbert submodules of $H^{2}(\mathbb{D})$ for $\mathbb{Z}^{+} \times_{\alpha} \mathcal{A}(\mathbb{D})$ (when $\alpha$ is an irrational rotation). One is tempted to ask about generalizations of Lemma 3.1 Theorem 4.2 is one such possible generalization.

Lemma 4.1. The algebra $\mathfrak{B}_{s c}$ of Lemma 3.1 contains the projection $P_{m}$ of $L^{2}(\mathbb{T})$ onto the subspace spanned by $z^{m} \forall m \in \mathbb{Z}$.

Proof. We first show $P_{-1} \in \mathfrak{B}_{s c}$. Define $C_{-1}=\bar{\mu} C_{\bar{\mu}}$ and $A_{k}=1 / k \sum_{j=1}^{k} C_{-1}^{j}$ for $k \geq 1$. Let

$$
S=\left\{f \in L^{2}(\mathbb{T}): \lim _{k \rightarrow \infty}\left(A_{k}-P_{-1}\right) f=0\right\} .
$$

Note that $\lim _{k \rightarrow \infty}\left(A_{k}-P_{-1}\right) z^{-1}=0$. Also, if $i \neq-1$, then

$$
\begin{aligned}
\lim _{k \rightarrow \infty}\left(A_{k}-P_{-1}\right) z^{i} & =\lim _{k \rightarrow \infty}\left(\frac{1}{k} \sum_{j=1}^{k} \bar{\mu}^{(i+1) j} z^{i}\right) \\
& =\int_{\mathbb{T}} z^{i} d m(z) \\
& =0
\end{aligned}
$$

by the ergodic theorem. Since $S$ is a closed subspace of $L^{2}(\mathbb{T})$ containing $z^{i} \forall i \in \mathbb{Z}$, it follows that $S=L^{2}(\mathbb{T})$ and $P_{-1} \in \mathfrak{B}_{s c}$.

We now show that $P_{m} \in \mathfrak{B}_{s c}$ for $m \neq-1$. Fix $m \neq-1$. Define $C_{m}=\bar{\mu}^{-m} C_{\bar{\mu}}$ and $A_{k}=1 / k \sum_{j=1}^{k} C_{m}^{j}$ for $k \geq 1$. Let

$$
S=\left\{f \in L^{2}(\mathbb{T}): \lim _{k \rightarrow \infty}\left(A_{k}\left(I-P_{-1}\right)-P_{m}\right) f=0\right\} .
$$


Since $m \neq-1,\left(A_{k}\left(I-P_{-1}\right)-P_{m}\right) z^{m}=1 / k \sum_{j=1}^{k} \bar{\mu}^{-m j} \bar{\mu}^{m j} z^{m}-z^{m}=0$. If $i=-1$ (so that $i \neq m)$, then $\left(A_{k}\left(I-P_{-1}\right)-P_{m}\right) z^{i}=0$. If $i \neq-1$ and $i \neq m$, then

$$
\begin{aligned}
\left(A_{k}\left(I-P_{-1}\right)-P_{m}\right) z^{i} & =\frac{1}{k} \sum_{j=1}^{k} \bar{\mu}^{(i-m) j} z^{i} \\
& \rightarrow \int_{\mathbb{T}} z^{i} d m(z) \\
& =0
\end{aligned}
$$

by the ergodic theorem. Hence, since $S$ is a closed subspace of $L^{2}(\mathbb{T})$ containing $z^{i} \forall i \in \mathbb{Z}, S=L^{2}(\mathbb{T})$ and $P_{m} \in \mathfrak{B}_{s c}$ as $A_{k}\left(I-P_{-1}\right) \in \mathfrak{B}_{s c} \forall k \geq 1$.

Theorem 4.2. Consider $\mathfrak{B}_{\alpha}$ as an operator algebra on $L^{2}(\mathbb{T})$. The nontrivial invariant subspaces $\mathcal{M}$ of $L^{2}(\mathbb{T})$ under $\mathfrak{B}_{\alpha}$ are of the form $\mathcal{M}=z^{m_{0}} H^{2}(\mathbb{D})$ for $m_{0} \in \mathbb{Z}$.

Proof. Let $(0) \neq \mathcal{M} \subseteq L^{2}(\mathbb{T})$ be a closed invariant subspace under $\mathfrak{B}_{\alpha}$ and hence under $\mathfrak{B}_{s c}$. Then $P_{m} \mathcal{M} \neq(0)$ for some $m \in \mathbb{Z}$ and $\mathcal{M}$ contains $z^{m}$ for some $m \in \mathbb{Z}$. Define $m_{0}=\inf \left\{m \in \mathbb{Z}: z^{m} \in \mathcal{M}\right\}$. Note that if $m_{0}=-\infty$, then the invariance of $\mathcal{M}$ under $M_{z}$ yields $\mathcal{M}=L^{2}(\mathbb{T})$. If $m_{0} \neq-\infty$, we claim that $\mathcal{M}=$ $z^{m_{0}} H^{2}(\mathbb{D})$. The invariance of $\mathcal{M}$ under $M_{z}$ yields $z^{m_{0}} H^{2}(\mathbb{D}) \subseteq \mathcal{M}$. Then, let $f(z)=\sum_{-\infty}^{\infty} a_{n} z^{n} \in \mathcal{M}$ so that $P_{k}(f(z))=a_{k} z^{k} \in \mathcal{M} \forall k \in \overline{\mathbb{Z}}$ by Lemma 4.1] But then $a_{k}=0$ for $k<m_{0}$ by the definition of $m_{0}$. Hence, $f(z)=\sum_{n=m_{0}}^{\infty} a_{n} z^{n}=$ $z^{m_{0}} \sum_{n=0}^{\infty} a_{n+m_{0}} z^{n} \in z^{m_{0}} H^{2}(\mathbb{D})$.

\section{REFERENCES}

[Arv] W.B. Arveson, Subalgebras of $C^{*}$-algebras, Acta Math. 123 (1969), 141-224. MR 40:6274

[Bus] Dale R. Buske, Hilbert Modules over Semicrossed Products of the Disk Algebra, Dissertation, Iowa State University, Ames, 1997.

[BP] Dale R. Buske and Justin R. Peters, Semicrossed Products of the Disk Algebra: Contractive Representations and Maximal Ideals, Pacific J. of Math., Vol. iii, No. i, (1998), 55-71.

[DP] R.G. Douglas and V. Paulsen, Hilbert Modules over Function Algebras, Pitman Research Notes in Mathematics Series 217, Longman Scientific and Technical, Harlow, Essex, UK, 1989. MR 91g:46084

[Hof] K. Hoffman, Banach Spaces of Analytic Functions, Prentice- Hall, Englewood Cliffs, NJ, 1962. MR 24:A2844

[HPW] T. Hoover, J. Peters, and W. Wogen, Spectral Properties of Semicrossed Products, Houston J. Math. 19 (1993), no. 4, 649-660. [MR 94k:47067]

[MS] Paul S. Muhly and Baruch Solel, Hilbert Modules over Operator Algebras, Mem. Amer. Math. Soc. 117 (1995), no. 559. MR 96c:47060

[NRW] Eric Nordgren, Peter Rosenthal, and F.S. Wintrobe, Invertible Composition Operators on $H^{p}$, J. Funct. Anal. 73 (1987), 324-344. MR 89c:47044

$[\mathrm{Sz}-\mathrm{NF}]$ B. Sz. Nagy and C. Foias, Harmonic Analysis of Operators on Hilbert Space, NorthHolland, Amsterdam, 1970. MR 43:947

[Pow] S.C. Power, Completely contractive representations for some doubly generated antisymmetric operator algebras, Proc. Amer. Math. Soc. 126 (1998), pp. 2355-2359. MR 98k:47091

[Suc] I. Suciu, On the semi-groups of isometries, Studia Mat. 30 (1968), 101-110. MR 37:4671

Department of Mathematics, St. Cloud State University, St. Cloud, Minnesota 56301

E-mail address: dbuske@stcloudstate.edu 